\title{
DNA vaccines against the human papillomavirus type 16 E6 or E7 oncoproteins
}

\author{
Anthony P Wlazlo, ${ }^{1, a}$ Hongying Deng, ${ }^{1, b}$ Wynetta Giles-Davis, ${ }^{1}$ and Hildegund CJ Ertl ${ }^{1}$
}

${ }^{1}$ The Wistar Institute, 3601 Spruce St., Philadelphia, Pennsylvania 19104, USA.

DNA vaccines expressing the E6 or E7 oncoproteins of human papilloma virus type 16 (HPV-16) in either their wild-type form or fused to sequences that affect intracellular trafficking were tested for induction of protective immunity against tumor cell challenge in two models based on BALB/C and C57BI/6 mice. The DNA vaccines to E7 gave uniformly disappointing results, while the DNA vaccine that expressed $\mathrm{E} 6$ linked to a viral leader sequence protected BALB/c mice against tumor cell challenge given before or after vaccination. The efficacy of this vaccine could be enhanced by a DNA vector prime/viral vector boost regimen. In contrast, priming of mice with the DNA vaccines to E7 reduced the efficacy of a viral vector expressing the same antigen.

Cancer Gene Therapy (2004) 11, 457-464. doi:10.1038/sj.cgt.7700723

Published online 30 April 2004

Keywords: DNA; vaccine; papillomavirus; cancer; E6; E7

$\mathrm{C}$ ervical cancer is most commonly a consequence of sexually transmitted, persistent infections with oncogenic types of HPVs, most notably HPV-16. ${ }^{1}$ Cervical cancer is the second most common cause of cancer death in women worldwide, claiming approximately 300,000500,000 lives each year. Upon sexual transmission, HPV16 (and other oncogenic types of sexually transmitted HPVs) infects the basal and parabasal cells of the cervical mucosa where transcription and translation of the early antigens of HPV-16 leads to cellular atypia followed by preinvasive neoplasia. ${ }^{2}$ Cellular transformation is initiated by the three viral oncoproteins, E5, E6 and E7, which cause dysregulation of cell cycle control. ${ }^{2,3}$ The oncoproteins of HPV-16 being causative for transformation of HPV-16-infected cells are thereby natural targets for active immunotherapy of HPV-16-associated cervical cancer.

A number of vaccine modalities to HPV-16 oncoprotein-expressing tumor cell lines have been tested in preclinical animal models focusing mainly on vaccines to E7, ${ }^{4-10}$ although studies with vaccines to E6 ${ }^{4,7,10}$ and $\mathrm{E} 5^{11}$ have also been reported. Our vaccine effort initially focused on viral recombinant vaccines expressing the E6 or E7 protein. They showed that E1-deleted adenoviral vectors to $\mathrm{E} 7$ induce potent and tumor-protective $\mathrm{CD} 8^{+}$

\footnotetext{
Received September 17, 2003.

Address correspondence and reprint requests to: Dr Hildegund CJ Ertl, The Wistar Institute, 3601 Spruce St., Philadelphia, PA 19104, $U S A$.

E-mail: ertl@wistar.upenn.edu

${ }^{\text {a } C u r r e n t ~ a d d r e s s: ~ N I H, ~ N I A I D, ~ L I D, ~ R e s p i r a t o r y ~ V i r u s e s ~ S e c t i o n, ~}$ 50 South Drive MSC-8011, Bethesda, MD, 20892, USA.

${ }^{\mathrm{b}}$ Current address: Howard Hughes Medical Institute, University of Pennsylvania, Philadelphia, PA 19104, USA.
}

T-cell responses in $\mathrm{C} 57 \mathrm{Bl} / 6$ mice. In contrast, $\mathrm{BALB} / \mathrm{c}$ mice displayed partial $\mathrm{CD}^{+}{ }^{+}$T-cell-mediated protection induced by vaccinia or adenoviral recombinant vaccines expressing the E6 of HPV-16 but could not be protected by the vaccines to E7. ${ }^{7}$

In this report, we extended our studies to DNA vaccines expressing the E7 or E6 proteins of HPV-16. In addition to those expressing wild-type versions of the oncoproteins, we also tested DNA constructs that express the oncoproteins linked to various polypeptides that affect intracellular trafficking. In one construct, the oncoproteins were fused to a viral leader sequence that prevents nuclear localization and instead affects secretion. A second modification involved addition of a leader sequence and the lysosome-associated membrane protein (LAMP)-1, which channels proteins upon secretion into lysosomes resulting in improved association with $\mathrm{MHC}$ class II determinants. This modification, used previously for HPV-16 E7 vaccines, was shown to also improve the induction of $\mathrm{MHC}$ class I-restricted $\mathrm{CD}^{+}$T-cell responses. ${ }^{5,12}$ Lastly, we expressed E6 and E7 linked to ubiquitin that promotes proteolytic degradation of proteins, thus increasing the pool of peptides available for transport into the endoplasmic reticulum where binding to MHC class I occurs. ${ }^{13}$ Our results showed poor efficacy of DNA vaccines expressing E7 in a C57B1/6 subcutaneous tumor cell challenge model. In contrast, DNA vaccines expressing E6 linked to a leader sequence provided protective immunity in the BALB/c tumor cell challenge model both if given prophylactically or therapeutically. In $\mathrm{BALB} / \mathrm{c}$ mice, but not in $\mathrm{C} 57 \mathrm{Bl} / 6$ mice, vaccine efficacy could be improved by DNA vaccine priming followed by a booster immunization with a low dose of an E1-deleted viral recombinant vector expressing the corresponding antigen. 


\section{Materials and methods}

\section{Animals}

Female 6-8-week-old BALB/c mice (H-2d) and C57Bl/6 mice (H-2b) (Taconic, Germantown, NY) were used for all experiments. Mice were housed at The Animal Facility of The Wistar Institute. Rats were purchased from Harlane Sprague-Dawley (Indianapolis, IN) and used at 3 months of age.

\section{Cell lines}

The following cell lines were used throughout the experiments: 293 cells were used for propagation of adenoviruses. COS-7 cells were utilized for transfection studies. The $\mathrm{t}(10) 1$ rasE7E6 cell line is derived from a primary embryonal BALB/c fibroblasT-cell line spontaneously immortalized due to the loss of both p53 alleles and transformed by transfection with a triple promoter retroviral vector expressing v-Ha-ras and E6 and E7 of HPV-16. ${ }^{7}$ The TC-1 cell line (provided by TC Wu, John Hopkins, Baltimore, MD) originated from lung epithelia cells immortalized by transfection with the v-Ha-ras, E6 and E7 genes of HPV-16.5 All of the above-described cell lines were grown in Dulbecco's modified Eagle's medium (DMEM, Life Technologies, Rockville, MD) supplemented with $10 \%$ fetal bovine serum (FBS), HEPES buffer and antibiotics. HT-2 cells, which are dependent on IL-2 or IL-4 for survival and proliferation were grown in DMEM supplemented with $10 \% \mathrm{FBS}, 10^{-6} \mathrm{M}$ 2-mercaptoethanol and $10 \%$ rat concanavalin A supernatant.

\section{Expression vectors}

DNA vaccine constructs. All DNA vaccines are based on a modified version of the expression vector pVR1012.2. containing the cytomegalovirus (CMV) promoter (generously provided by Vical, Inc., San Diego, CA). The kanamycin-resistance gene was removed from the vector and replaced with the ampicillin-resistance gene. The ampicillin-resistance gene has two strong $\mathrm{CpG}$ motifs that are active in mice. ${ }^{14}$ Constructs pVRE6, pVRE7, pVRLDE6 and pVRLDE7 have been previously described. $^{15}$ The plasmids pVRLDE6 and pVRLDE7 express the E19 leader sequence of the human adenovirus (AdHu) serotype 2 fused to the amino terminus of the HPV-16 oncoproteins.

Constructs pVRE6LAMP-1 and pVRE7LAMP-1 were generated from pre-existing constructs (pCVMSigE6LAMP-1 and pCVMSigE7LAMP-1). Specific oligonucleotides (CyberSyn, Lenni, PA) were generated based on the sequence for the signal peptide and the LAMP-1 cytoplasmic domain ${ }^{12}$ containing the $X b a \mathrm{I}$ and Bam HI restriction sites $\left(5^{\prime}\right.$ and $3^{\prime}$ ends respectively). The preexisting constructs were used as templates for a standard polymerase chain reaction (PCR). The PCR fragment was digested with $\mathrm{Xba \textrm {I }}$ and $\mathrm{BamHI}$ and ligated into similarly digested pVR1012.2. These constructs express the E6 and E7 oncoproteins fused to the signal sequence and the integral membrane sequence of the LAMP-1 protein.
To generate constructs pVRE6UBI and pVRE7UBI, the pVRE6 and pVRE7 vectors were digested with $X b a \mathrm{I}$ and BamHI enzymes. The liberated oncogenes were ligated into pVRp53UBI from which the p53 insert had been removed using the same restriction enzymes. ${ }^{16}$ The final constructs express the ubiquitin protein fused to the amino terminus of the HPV-16 oncoproteins.

Correct insertion of the fragments was verified by sequencing through the Genomics Facility of The Wistar Institute.

Adenoviral expression constructs. E1-deleted replication-defective human serotype 5 adenovirus recombinants expressing either the E6 or E7 genes of HPV $-16^{7}$ or the L1 of HPV $-16^{17}$ have been described previously.

Recombinant baculovirus expression constructs. Baculovirus recombinants to HPV-16 E7 and E6 were used to generate oncoproteins as described previously. ${ }^{15}$

Expression. of baculovirus recombinant proteins and their purification. Recombinant baculovirus was generated by the Vector Core Facility of The Wistar Institute by cotransfection of the transfer vector with BaculoGold DNA (BD, Pharmingen, Torre Pines, CA) into Sf9 insect cells as described. ${ }^{18}$ For protein production, Sf9 cells $\left(500 \mathrm{ml}: 5 \times 10^{6}\right.$ cells $\left./ \mathrm{ml}\right)$ were infected at a multiplicity of infection of 10 with the various baculovirus recombinants for 72 hours. Cells were then harvested and freeze/thawed $3 \times$ in PBS. Following low-speed clarification, cell debrisfree supernatants were precipitated with ammonium sulfate. Precipitated proteins were aliquoted and protein content was determined by the Bradford method using a Bio Rad protein assay (Bio Rad, Hercules, CA)

\section{Propagation and purification of viruses}

Adenovirus recombinants were propagated on the E1transfected 293 cell line. Semiconfluent monolayers of cells were infected with 0.1 plaque forming units (PFU)/ cell of virus. Cells were harvested after 3 days and freeze/ thawed three times. Cell debris was removed by centrifugation at 2000 r.p.m for 30 minutes. Virus was titrated on 293 cell monolayers. Cells plated in 6-well plates were infected with serial dilutions of virus-containing medium. The following day cells were overlaid with agarose. Viral plaques were counted 4-8 days later.

\section{Immunization of mice}

DNA vaccines $(50-100 \mu \mathrm{g})$ were injected into mice intramuscularly (i.m. ) in both quadricep muscles, dividing the dose equally to reduce variability. Mice were boosted once with the same DNA vaccine $(50-100 \mu \mathrm{g}) 3$ weeks after the initial injection. Some mice were further boosted subcutaneously (s.c.) 2-3 weeks later with $10^{6}$ PFU of replication-defective adenovirus expressing the E6, the E7 or the L1 of HPV-16. At 3 weeks after the DNA boost or 2 weeks after the viral boost, mice were challenged s.c. with $3 \times 10^{4} \mathrm{t}(10) 1$ rasE7E6 cells or with $2 \times 10^{5}$ TC- 1 cells. After tumor cell challenge, mice were 
checked three times/week. They were scored as tumor bearing when the s.c. growth exceeded $2 \mathrm{~mm}$ in diameter. Mice were euthanized once tumors reached a size of $\sim 1 \mathrm{~cm}$ in diameter. Mice were depleted of NK cells with a rabbit serum against asialo-GM1 ganglioside (Wako Inc., Wako, TX) as described. ${ }^{19}$

\section{In vitro expansion of splenocytes}

Splenocytes were harvested 2 weeks after immunization and cultured at $6 \times 10^{6}$ cells in $1.6 \mathrm{ml}$. of DMEM (with $2 \%$ FCS, HEPES buffer, $10 \mathrm{~mm}$ 2-mercaptoethanol, nonessential amino acids, glutamine, sodium pyruvate and antibiotics). Adenoviral recombinants (0.1 PFU/cell) or baculovirus produced protein $(1 \mathrm{~g} / \mathrm{ml})$ were added to each well.

\section{Intracellular cytokine staining}

Freshly harvested splenocytes were incubated with $1 \mu \mathrm{g}$ / $\mathrm{ml}$ of the peptide carrying the immunodominant $\mathrm{H}-2 \mathrm{~b}$ MHC class I epitopes of E7 (RAHYNIVTF) or a p53 control peptide for 5 hours in 96-well U-bottom plates (Costar, Cambridge, MA) at a concentration of $10^{6}$ cells/ well in a volume of $200 \mu \mathrm{l}$ complete DMEM supplemented with $50 \mathrm{U} / \mathrm{ml}$ of human recombinant IL- 2 and $1 \mu \mathrm{l} / \mathrm{ml}$ of Brefeldin A (Golgistop, Pharmingen, La Jolla, $\mathrm{CA}^{20}$ ). After 5 hours, the cells were washed and surface-stained with an FITC-conjugated rat anti-mouse CD8 antibody (Pharmingen, La Jolla, CA) followed by fixation/permeabilization (Cytofix/Cytoperm, Pharmingen) and intracellular staining for IFN- $\gamma$ (PE-conjugated rat anti-mouse IFN- $\gamma$, Pharmingen) according to the manufacturer's instructions. After washing, cells were examined by twocolor flow cytometry using an EPICS Elite XL (BeckmanCoulter, Inc., Miami, FL) and data were analyzed using the WinMDi software.

\section{Cytokine-release assay}

IL-2 secretion was tested for by culturing $6 \times 10^{6}$ splenocytes of immune or sham-vaccinated mice in $1.6 \mathrm{ml}$. DMEM supplemented with $2 \%$ FCS and $10^{-6} \mathrm{M}$ 2-mercaptoethanol. Baculovirus-derived E6 or E7 protein was added to a final concentration of $1 \mu \mathrm{g} / \mathrm{ml}$. Supernatants were harvested 24 hours later and tested for induction of proliferation of the IL-2-dependent HT-2 indicator cell line as described. ${ }^{21}$

\section{Results \\ Validation of DNA constructs}

Construction of pVRE6, pVRE6LD, pVRE6LAMP-1, pVRE6UBI, pVRE7, pVRE7LD, pVRE7LAMP-1 and pVRE7UBI were derived as described in Material and methods. The inserts were sequenced and transcript expression was confirmed by a reverse transcription PCR. The different vectors including the viral vectors used for vaccination are listed in Table 1.
Table 1 List of vectors used for vaccination

\section{E1-deleted adenoviral vectors AdHu5E6 \\ AdHu5E7 \\ AdHu5L1 \\ Wild-type E6 sequence Wild-type E7 sequence Wild-type L1 sequence}

\begin{abstract}
${ }^{a}$ In the pVR1012.2 vector used for all of the constructs, the original kanamycin-resistance gene was replaced with an ampicillin-resistance gene.
\end{abstract}

\title{
Preventative DNA vaccination
}

Vaccines were tested for efficacy in two transplantable tumor models in $\mathrm{C} 57 \mathrm{Bl} / 6$ mice and in $\mathrm{BALB} / \mathrm{c}$ mice using two transplantable tumor models based on TC-1 and $\mathrm{t}(10) 1$ rasE6E7 cells originated from $\mathrm{C} 57 \mathrm{Bl} / 6$ and $\mathrm{BALB} / \mathrm{c}$ mice, respectively. Both tumor cell lines express E6 and E7 of HPV-16 together with v-Ha-ras. After two immunizations with DNA vaccines expressing the different forms of the E6 or E7 oncoproteins, mice were challenged s.c. with tumor cells, that is, $\mathrm{t}(10) 1 \mathrm{rasE7E6}$ $(\mathrm{BALB} / \mathrm{c})$ or $\mathrm{TC}-1(\mathrm{C} 57 \mathrm{Bl} / 6)$ cells, and monitored for tumor formation. Mice were scored as tumor bearing when tumors reached $\sim 2 \mathrm{~mm}$ in diameter. Greater than $90 \%$ of mice receiving an empty vector alone developed tumors after challenge (Fig 1). The DNA vaccines expressing wild-type E6, or E6 fused to LAMP-1 or ubiquitin protected a small percentage $(\sim 20 \%)$ of BALB/ c mice (Fig 1a). The vaccine expressing E6 linked to a viral leader sequence (pVRE6LD) protected $\sim 70 \%$ of the animals against tumor development and delayed onset of tumors in those mice that remained susceptible to tumor challenge. In $\mathrm{C} 57 \mathrm{Bl} / 6$ mice, none of the E6-expressing vaccines induced significant protection against challenge with the syngeneic TC-1 tumor cell line (Fig 1c). The DNA vaccines expressing E7 were also largely ineffective in BALB/c (Fig 1b) or C57Bl/6 (Fig 1d) mice. Only the construct expressing $\mathrm{E} 7$ linked to ubiquitin provided some protection $(\sim 20 \%)$ to $\mathrm{BALB} / \mathrm{c}$ mice, while the pVRE7LAMP-1 vaccine provided protection to $\sim 50 \%$ of $\mathrm{C} 57 \mathrm{Bl} /$ 6 mice. Nevertheless, neither of these results reached statistical significance. 




Figure 1 Efficacy of preventative DNA vaccination. BALB/c or $\mathrm{C} 57 \mathrm{BI} / 6$ mice were vaccinated i.m. twice with $100 \mu \mathrm{g}$ of E6 and E7expressing vectors. At 3 weeks after the final vaccination, mice were challenged s.c. with either $3 \times 10^{4} \mathrm{t}(10) 1 \mathrm{rasE} 7 \mathrm{E} 6$ cells (BALB/c mice) or $2 \times 10^{5} \mathrm{TC}-1$ cells (C57BI/6 mice). (a) BALB/c mice vaccinated with DNA vaccines to $E 6$, (b) BALB/c mice vaccinated with DNA vaccines to $\mathrm{E} 7$, (c) $\mathrm{C} 57 \mathrm{BI} / 6$ mice vaccinated with DNA vaccines to $\mathrm{E} 6$, (d) C57BI/6 mice vaccinated with DNA vaccines to E7. X-mice vaccinated with the insert-free pVR1012.2 vector. Number of animals: (a) 29, (b) 5, (c) 26, (d) 17. Open squares-mice vaccinated with DNA vaccines expressing wild-type E6 or E7: (a) 28, (b) 5, (c) 28, (d) 17. Closed squares-mice vaccinated with DNA vaccines expressing E6 or E7 linked to a viral leader sequence (a) 18, (b) 5, (c) 29, (d) 15. Squares with cross-mice immunized with DNA vaccines modified to express E6 or E7 linked to a signal sequence and LAMP: (a) 8 , (b) 5, (c) 5, (d) 10 . Half-open squaresmice immunized with DNA vaccines expressing E6 or E7 linked to ubiquitin: (a) 8 , (b) 5, (c) 5, (d) 10. The graph shows the combined results of several experiments using the number of mice indicated above. Statistical significance of \% tumor-free survival and of the kinetics of onset of tumors was calculated by Student's $t$-test. Data with $P$-values $<.05$ were considered significant. *Significant increase in \% tumor-free survival. ${ }^{*}$ Significant delay in onset of visible tumors.

\section{DNA prime/viral recombinant boost vaccination}

The immune response to DNA vaccines can be augmented by booster immunization with a heterologous vaccine carrier such as a viral vector expressing the same antigen. ${ }^{22,23}$ As neither of the DNA vaccines induced complete protection against tumor cell challenge in $\mathrm{BALB} / \mathrm{c}$ or $\mathrm{C} 57 \mathrm{Bl} / 6$ mice, we tested whether boosting of DNA vaccine primed mice with a low dose of an E1deleted adenoviral recombinant expressing the corresponding antigen augmented resistance to tumor cell challenge. In these experiments, BALB/c mice were tested using DNA vaccines expressing wild-type E6 or E6 linked to a leader sequence (Fig 2a), while C57Bl/6 mice were tested with the entire set of DNA vaccines to E6 (Fig 2b)
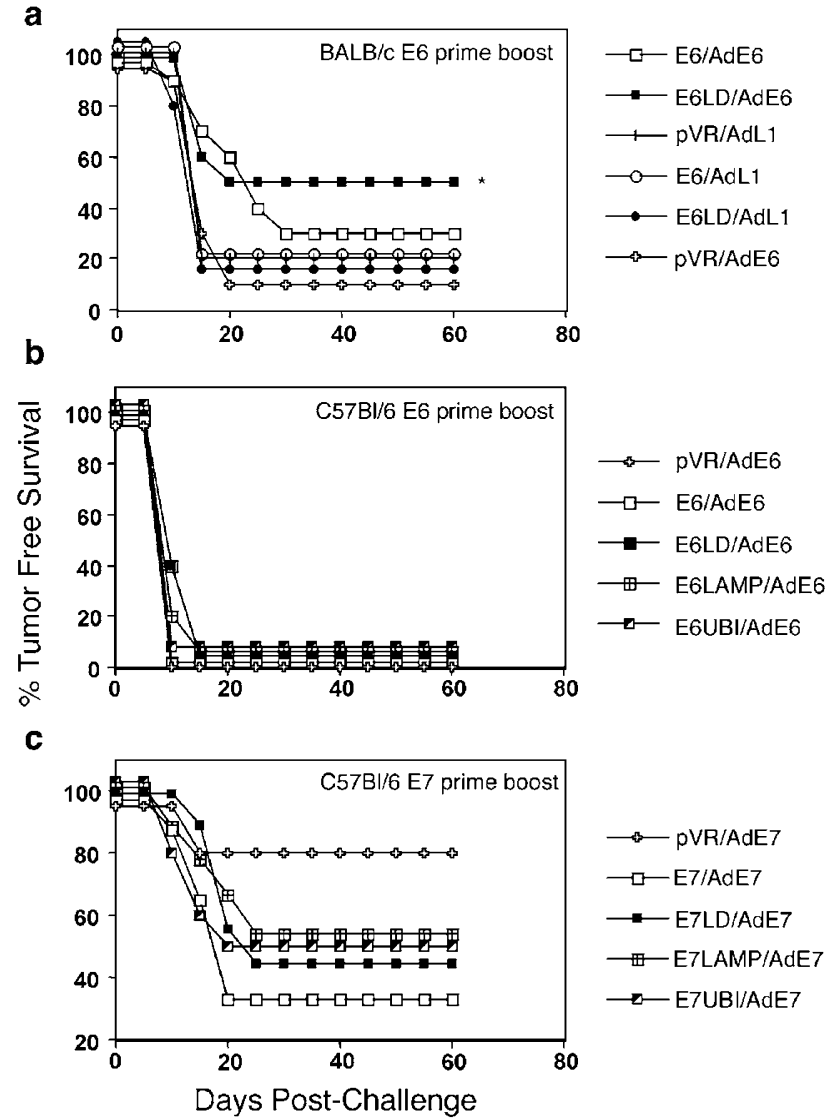

Figure 2 Efficacy of a DNA vaccine prime-adenoviral recombinant boost regimen. $\mathrm{BALB} / \mathrm{c}$ or $\mathrm{C} 57 \mathrm{BI} / 6$ mice were vaccinated i.m. twice with $100 \mu \mathrm{g}$ of the DNA vaccines. At 3 weeks after the second dose of DNA, mice were boosted s.c. with either $10^{6} \mathrm{PFU}$ of AdHu5E7 or AdHu5E6 virus. At 7 days following viral recombinant boost, mice were challenged with either $3 \times 10^{4} t(10) 1$ rasE7E6 cells (BALB/c) or $2 \times 10^{5} \mathrm{TC}-1$ cells $(\mathrm{C} 57 \mathrm{BI} / 6)$ s.c. (a) BALB/c mice vaccinated with vaccines to E6: open squares-mice primed with the pVRE6 vector and boosted with the AdHu5E6 virus; $n$ (number of animals) $=15$. Closed squares-mice primed with the pVRE6LD vector and boosted with the AdHu5E6 virus; $n=15$. Open crosses-mice primed with the insert-free pVR1012.2 control vector and boosted with AdHu5E6 virus; $n=15$. Open circles-mice primed with the pVRE6 vaccine and boosted with the AdHu5L1 vaccine; $n=5$. Closed circles-mice primed with the pVRE6LD vaccine and boosted with the AdHu5L1 vaccine; $n=5$. Closed crosses-mice primed with the insert-free pVR1012.2 vector and boosted with the AdHu5L1 vaccine; $n=5$. (b) C57BI/6 mice immunized with vaccines to $\mathrm{E} 6$ and (c) $\mathrm{C} 57 \mathrm{BI} / 6$ mice immunized with vaccines to $\mathrm{E} 7$ : open crosses-mice immunized with the insert-free pVR1012.2 vector and boosted with the AdHu virus expressing E6 or E7; number of animals: $n=5$ (b) and 10 (c). Open squares-mice immunized with pVRE6 or pVRE7 and boosted with the corresponding AdHu5 vector, $n=5$ (b) and 8 (c). Closed squares-mice immunized with pVRE6LD or pVRE7LD and boosted with the corresponding AdHu5 vector, $n=5$ (b) and 9 (c). Squares with cross-mice immunized with pVRE6LAMP or pVRE7LAMP and boosted with the corresponding AdHu5 vector $n=5$ (b) and 9 (c). Half-open squares-mice immunized with pVRE6UBI or pVRE7UBI and boosted with the corresponding AdHu5 vector, $n=5$ (b) and 10 (c). Data were collected as described in the legend to Figure 1. 
or E7 (Fig 2c). Priming of BALB/c mice with the pVRE6LD vaccine followed by booster immunization with the AdHu5E6 vaccine provided a statistically significant increase in protection compared to priming with an empty DNA vector prior to immunization with the AdHu5E6 vaccine. None of the C57Bl/6 mice immunized with DNA vaccines to E6 followed by a booster immunization with the AdHu5E6 vaccine were protected. The E7 vaccines had a paradoxical effect in $\mathrm{C} 57 \mathrm{Bl} / 6$ mice. In this model, priming with any of the DNA vaccines to E7 impaired the efficacy of the AdHu5E7 vaccine that upon immunization of control vector-primed mice protected most, that is, $80 \%$, of the animals while priming with E7-expressing DNA vaccines reduced the level of protection to $\leqslant 50 \%$.

\section{Therapeutic immunization}

Considering the nature of HPV-16 infections and the sequence of expression of the different viral antigens, vaccines to the viral oncoprotein should be used therapeutically rather than for prevention of an infection, which can best be achieved by vaccines inducing neutralizing antibodies to the viral surface antigen L1. ${ }^{24}$ We tested if the most effective of our DNA vaccines, that is, the pVRE6LD vaccine could prevent tumor progression if given after challenge in $\mathrm{BALB} / \mathrm{c}$ mice. Naïve BALB/c mice were injected with $10^{4} \mathrm{t}(10) 1 \mathrm{rasE7E6}$ cells s.c. After 3 days, the same mice were immunized i.m. with $100 \mu \mathrm{g} /$ mouse of the pVRE6LD vaccine or the insertfree vector. Mice were boosted with $100 \mu \mathrm{g}$ of DNA/ mouse 7 days after the initial DNA vaccination. The pVRE6LD vaccine caused tumor regression in $\sim 80 \%$ of the animals (Fig 3a). The empty vector achieved protection in $\sim 30 \%$ of the animals. To test if innate immune responses such as NK cells activated by the bacterial components of the vector ${ }^{25}$ rather than just E6-specific adaptive immune responses contributed to the therapeutic effects of the vaccine, mice were depleted of NK cells by treatment with an antiserum to asialo-GM1 ganglioside and then tested in a postchallenge vaccination experiment. As shown in Figure 3b, depletion of NK cells did not influence tumor development suggesting that this lymphocyte subset did not contribute to the postchallenge efficacy of the pVRE6LD vaccine.

\section{Mechanisms of protection}

Protection against HPV-16-associated tumors induced by vaccines to the viral oncoproteins is linked to the induction of cellular rather than humoral immunity. ${ }^{7}$ To test if the DNA vaccines induced detectable -cel T-cell responses to the transgene product, BALB/c mice were vaccinated with the pVRE6, the pVRE6LD or the insertfree vector or $\mathrm{C} 57 \mathrm{Bl} / 6$ mice were vaccinated with the entire set of DNA vaccines to E7. Splenocytes were harvested 3 weeks after the second vaccination and cultured with an irrelevant protein or with the viral oncoprotein expressed by the DNA vaccine, a procedure that activates primarily antigen-primed $\mathrm{CD}^{+}$T-cells. Supernatants were harvested 24 hours later and tested for

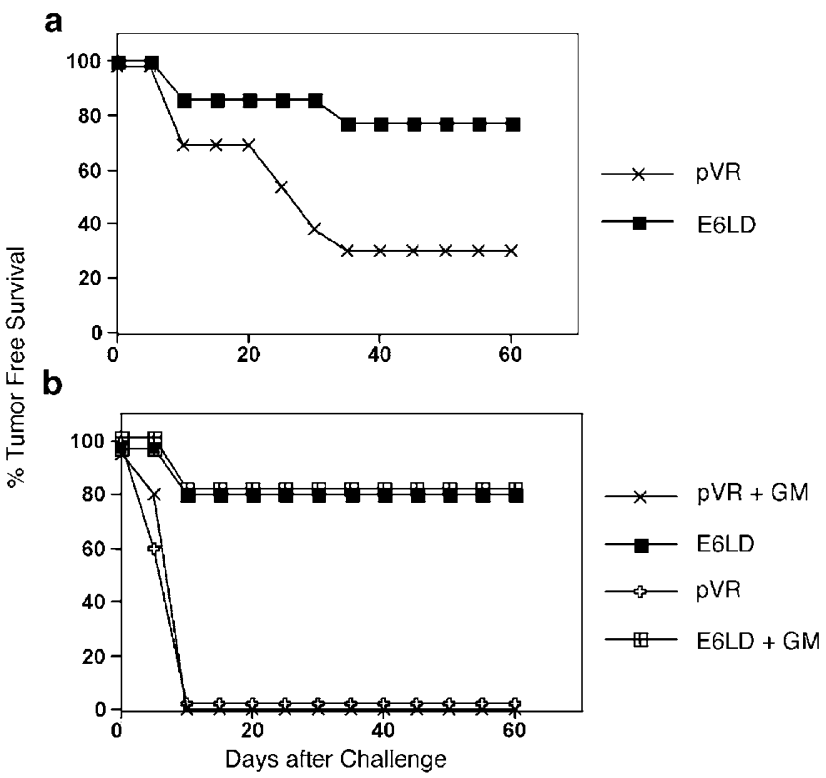

Figure 3 Efficacy of DNA vaccines given after tumor cell challenge. (a) BALB/c mice were challenged s.c. with $10^{4} \mathrm{t}(10) 1 \mathrm{rasE7E6}$ cells. At 3 and 10 days postchallenge, the mice were immunized i.m. with $100 \mu \mathrm{g}$ of insert-free pVR1012.2 vector $(\mathrm{X}, n=13)$, or pVRE6LD (closed squares, $n=8$ ). (b) mice treated with a depleting dose of a serum to NK cells as well as untreated control animals were challenged and vaccinated as described in Figure $4 a$. X-insert-free pVR1012.2; vector; open crosses-anti-NK treatment and + pVR1012.2; closed squares-pVRE6LD; squares with cross-antiNK treatment and + pVRE6LD.

IL-2 on the HT-2 indicator cells and for IL-4 and IFN- $\gamma$ by an ELISA. We were unable to demonstrate in any of the experiments significant release of either IFN- $\gamma_{-}$or IL4 in response to the vaccine antigen (data not shown). Secretion of low levels of IL-2 could be detected from splenocytes of mice vaccinated with the pVRE6 or the pVRE6LD vector (Fig 4a). Levels of secretion were comparable and thus not linked to the superior efficacy of the pVRE6LD vaccine. In $\mathrm{C} 57 \mathrm{Bl} / 6$ mice, none of the $\mathrm{E} 7$ expressing vaccines induced secretion of detectable amounts of cytokines (i.e., IFN- $\gamma$, IL-2 or IL-4) as shown in Figure $4 b$ for IL-2.

In addition, splenocytes were tested for $\mathrm{CD} 8{ }^{+} \mathrm{T}$-cell activity by a number of different techniques. We were unable to demonstrate significant lysis of HPV-16 oncoprotein expressing target cells by a traditional ${ }^{51} \mathrm{Cr}$ release assay using in vitro expanded splenocytes as effector cells. Stimulation of splenocytes for 5 hours with a peptide carrying the immunodominant epitope of E7 in presence of a drug that inhibits secretion of proteins revealed low frequencies (i.e., $<0.4 \%$ ) of E7-specific IFN- $\gamma$-producing CD8 ${ }^{+}$T-cells in mice immunized with the pVRE7LD or pVRE7LAMP-1 vaccines (Table 2). Frequencies were below those previously reported for AdHu5E7-vaccinated $\mathrm{C} 57 \mathrm{Bl} / 6$ mice $\left(0.8-1 \%{ }^{7}\right)$. They failed to increase upon challenge with $\mathrm{TC}-1$ cells (data not shown), which was also in contrast to our previous observation with mice vaccinated with a protective dose of the AdHu5E7 vaccine. ${ }^{20}$ 


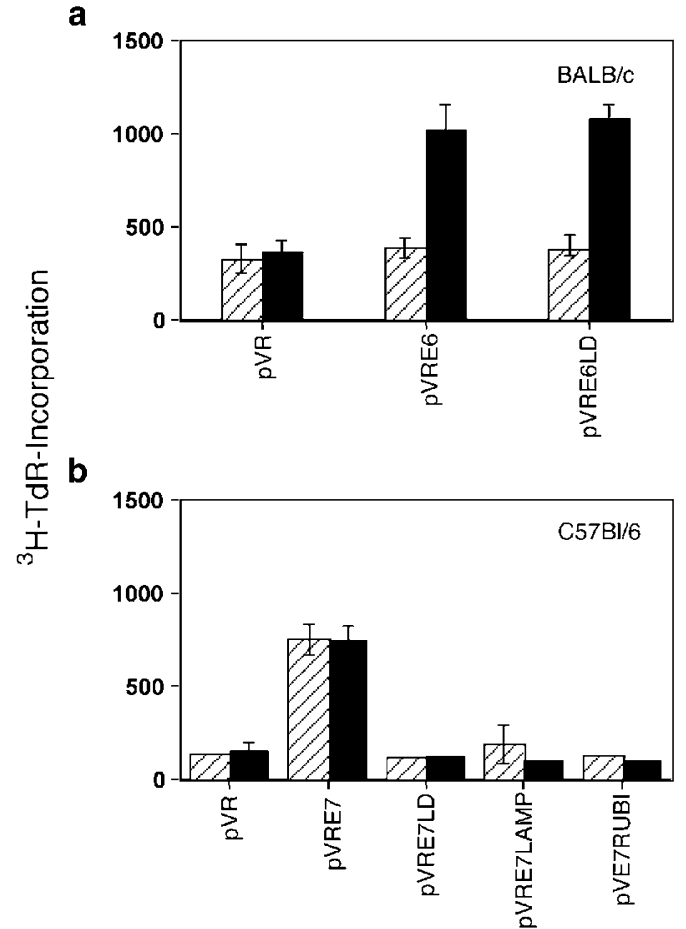

Figure 4 IL-2 release by splenocytes from DNA vaccinated mice. (a) $\mathrm{BALB} / \mathrm{C}$ or (b) $\mathrm{C} 57 \mathrm{BI} / 6$ mice were immunized twice with the DNA vaccines. At 3 weeks after the second immunization, splenocytes were cultured for 24 hours with E7 peptide (black bars) or a control peptide (striped bars). Cell-free supernatants were tested for induction of proliferation of the HT-2 cell line. Data show means of triplicate samples \pm standard deviations. TdR-thymidine.

Table 2 Frequency of IFN- $\gamma^{+} \mathrm{CD}^{+}$cells ${ }^{\mathrm{a}}$ E7 peptide

\begin{tabular}{lccc}
\hline & + & - & $\Delta$ Frequency $^{\text {b }}$ \\
\hline pVR1012.2 & 0.07 & 0.07 & 0 \\
PVRE7 & 0.07 & 0.12 & -0.05 \\
PVRE7LD & 0.52 & 0.15 & 0.37 \\
PVRE7LAMP-1 & 0.33 & 0.13 & 0.18 \\
PVRE7UBI & 0.08 & 0.12 & -0.04 \\
\hline
\end{tabular}

Groups of BALB/c mice were immunized three times in 2-week intervals with $100 \mu \mathrm{g}$ of DNA. At 3 weeks after the last injection, splenocytes were harvested and cultured with the control peptide $(-)$ or the E7 peptide (+) for 5 hours in the presence of Brefeldin. Cells were then stained for CD8 and intracellular IFN- $\gamma$.

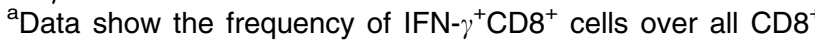
cells.

${ }^{\mathrm{b}}$ Frequency of IFN $-\gamma^{+} \mathrm{CD} 8^{+}$cells in cultures incubated with the E7 peptide minus frequencies of IFN $-\gamma^{+} \mathrm{CD} 8^{+}$cells in cultures incubated with the control peptide.

\section{Discussion}

We tested a set of DNA vaccines expressing either the E6 or E7 antigens of HPV-16 for induction of protection to challenge with HPV-16 E6/E7-expressing tumor cells in two different mouse models. We had shown previously, using viral recombinant vaccines, that is, E1-deleted adenoviral vectors derived from the human serotype 5 and vaccinia virus recombinants from the Copenhagen strain, that E1-deleted adenoviral vectors expressing E7 provide complete protection to $\mathrm{TC}-1$ cell challenge in C57Bl/6 mice, while both types of viral vectors expressing E6 provide partial protection (30-40\%) to BALB/c mice against challenge with the $t(10) 1$ rasE7E6 cell line. ${ }^{7}$ This discrepancy in protection was at least in part linked to genetic restriction of T-cell-mediated immune responses to the short oncoproteins of HPV-16. ${ }^{26}$ E7 carries an immunodominant epitope able to associate with MHC class I determinants of the $\mathrm{H}-2 \mathrm{~b}$ haplotype $\mathrm{e}^{27}$ but not of the $\mathrm{H}-2 \mathrm{~d}$ haplotype. ${ }^{7}$ The E7 protein lacks a functional epitope with high affinity to either $\mathrm{H}-2 \mathrm{~d}$ or $\mathrm{H}-2 \mathrm{~b}$ MHC class II determinant. ${ }^{7}$ Protection in $\mathrm{C} 57 \mathrm{Bl} / 6$ mice was consequently mediated by $\mathrm{CD} 8^{+} \mathrm{T}$-cells, which are best induced by E1-deleted adenoviral vectors. The E6 antigen lacks a $\mathrm{CD} 8^{+} \mathrm{T}$-cell epitope for either mouse haplotype but apparently carries an immunogenic MHC class II epitope for $\mathrm{BALB} / \mathrm{c}$ mice. ${ }^{7}$ In $\mathrm{BALB} / \mathrm{c}$ mice, protection was mediated by $\mathrm{CD} 4{ }^{+}$T-cells, which could be induced by vaccinia virus vectors as well as by E1-deleted adenoviral recombinants. Ir-gene control of T-cell responses thereby limited the usefulness of E6 and E7 viral recombinant vaccines in inbred strains of mice and one would expect genetically restricted efficacy in an outbred human population as well.

DNA vaccines, through yet to be defined mechanisms, have been shown to overcome Ir-gene control and to induce responses to proteins that are otherwise poorly immunogenic in certain MHC haplotypes if given by other vaccine carriers. ${ }^{28}$ To assess if we could expand the efficacy of vaccines to HPV-16 oncoproteins, we developed a set of DNA vaccines expressing either E6 or E7 in wild-type form or fused to sequences known to affect antigen trafficking, processing and presentation by MHC class I or class II determinants and tested these vaccines in both $\mathrm{BALB} / \mathrm{c}$ and $\mathrm{C} 57 \mathrm{Bl} / 6$ mice.

Our results with DNA vaccines to E6 yielded promising results in $\mathrm{BALB} / \mathrm{c}$ mice. The DNA vaccines expressing E6 linked to a viral leader sequence induced protection in the majority of mice if given before or even after tumor cell challenge. In the BALB/c model, protection is mediated by $\mathrm{CD}^{+}{ }^{+} \mathrm{T}$-cells and DNA vaccines expressing a secreted form of E6 outperformed viral recombinant vaccines, which as reported previously induce protective immunity in $\sim 30-40 \%$ of vaccinated BALB $/ \mathrm{c}$ mice. ${ }^{7}$ We showed previously that a DNA vaccine expressing a biologically inactive form of mouse p53 linked to a viral leader sequence induced better protection against a tumor cell line expressing high levels of p53 compared to DNA vaccines expressing other modifications of $\mathrm{p} 53 .{ }^{16}$ In this system, both $\mathrm{CD}^{+}{ }^{+}$and $\mathrm{CD} 8{ }^{+}$T-cells mediated protection. Addition of a leader sequence to proteins with nuclear localization domains such as p53 or E6 ensures cytoplasmic retention of the protein and in case of E6 channeling into the secretion pathway. Secretion of the 
protein may, through reprocessing, increase antigen presentation in association with MHC class II determinants and thus increase activation of antigen-specific CD4 ${ }^{+}$T-cells. This could not be confirmed experimentally as splenocytes from mice immunized either with the pVRE6 or the pVRE6LD vectors secreted negligible amounts of IL-4 and comparable levels of IL-2. Considering the intricacies of T-cell trafficking, lack of an in vitro T-cell-mediated recall response does not necessarily reflect lack of such a response in vivo. In both the p53 and the E6 tumor models, vaccines expressing the tumorassociated antigens linked to a leader sequence outperformed E1-deleted adenoviral vectors as well as poxvirus vectors. ${ }^{16}$ The result is surprising considering that viral vectors induce in general far more potent transgene product-specific immune responses compared with DNA vaccines. ${ }^{29}$ It is conceivable that epitopes with comparatively low affinity to MHC determinants may be more immunogenic if delivered by a DNA vaccine than by a viral recombinant vector. DNA vectors, unlike viral vaccine carriers, do not express any competing epitopes, which may help the induction of T-cells to low-affinity epitopes, which are displayed in limiting amounts by MHC-molecules on the cell surface. In the same vein, DNA vaccines are thought to provide long-lasting production of antigen that may also optimize the chances of low levels of $\mathrm{MHC}$-epitope complexes to attract the attention of T-cells with corresponding receptors.

The DNA vaccines expressing E7 yielded uniformly disappointing results upon i.m. administration of two or more doses (protection results are only shown for the twodose regimen) by providing limited protection to TC-1 cell challenge and by inducing only marginal $\mathrm{CD}^{+} \mathrm{T}$-cell responses to the immunodominant epitope of E7. Previous reports have shown that protective immunity to TC-1 cell challenge could be induced by a DNA vaccine expressing E7 fused to LAMP-1. ${ }^{30}$ In these studies, in contrast to ours, the DNA vaccine was delivered by gene gun immunization that generally favors a Th2 response rather than a Th1 response that is best suited to drive activation of $\mathrm{CD}^{+}{ }^{+}$T-cells. ${ }^{31}$ Accordingly, frequencies reported in these studies were even lower than those achieved with our immunization protocol with the pVRE7LAMP-1 construct. Nevertheless, protection against TC-1 cell challenge upon immunization of mice with a variety of DNA vaccines expressing modified E7 given alone or in conjunction with genetic adjuvant has been established. ${ }^{8,9,30}$ These contrasting results can best be explained by differences in cell numbers used for tumor cell challenge; we used a dose of $2 \times 10^{5}$ TC- 1 given s.c. This dose leads to rapid development of tumors; within 10 days tumors commonly reach a size that requires euthanasia of the animals. Previously published results were based on a challenge model in which mice are injected with $10^{4}$ tumor cells intravenously. ${ }^{30}$ In this type of challenge, only a fraction of the tumor cells become established in the lung and proliferate into nodules. ${ }^{32}$ The number of tumor cells that has to be eliminated by tumor antigen-specific $\mathrm{CD}^{+}$T-cells is thus fairly low, which obviously influences vaccine efficacy.
Others have shown that gene gun delivery of DNA vaccines to E7 resulted in enhanced tumor growth of an E6/E7-transformed mouse cell line given s.c. to $\mathrm{C} 57 \mathrm{Bl} / 6$ mice. $^{33}$ The DNA vaccination was shown to direct the immune response towards the Th2 pathway resulting in an immune response that was incompatible with tumor cell eradication. We obtained comparable results when we inoculated the DNA vaccines to E7 through the i.m. route and then boosted mice with a low dose of the E1-deleted AdHu5E7 vector. This type of prime boost regimen, previously described to enhance the efficacy of vaccines to other viral antigens, ${ }^{29}$ reduced the level of protection achieved with the AdHu5E7 vaccine against tumor cell challenge in the $\mathrm{C} 57 \mathrm{Bl} / 6$ mouse model presumably by impairing activation of a protective $\mathrm{CD} 8^{+} \mathrm{T}$-cell response.

Other vaccine modalities expressing E7, such as E1deleted adenoviral vectors, ${ }^{7}$ viral replicons ${ }^{10}$ or bacterial vaccine vehicles ${ }^{34}$ have been shown to induce more potent $\mathrm{CD}^{+}$T-cell responses and reliable protection to experimental tumor cell challenge than DNA vaccines to E7. Especially, the findings that DNA vaccines to E7 can increase tumor progression or subvert the efficacy of other vaccine modalities if used for priming, raise concern for the validity of such vaccines for use in humans.

\section{Acknowledgments}

This work was supported by grants from NCI and NIAID. Dr AP Wlazlo was a recipient of a fellowship from the Cancer Research Foundation of America and from NIH/NIAID. We thank the Commonwealth Universal Research Enhancement Program, Pennsylvania Department of Health.

\section{References}

1. Stone KM, Karem KL, Sternberg MR, et al. Seroprevalence of human papillomavirus type 16 infection in the United States. J Infect Dis. 2002;186:1396-1402.

2. zur Hausen H. Papillomavirus infections - a major cause of human cancers. Biochim Biophys Acta. 1996;1288:F55-F78.

3. Munger K, Howley PM. Human papillomavirus immortalization and transformation functions. Virus Res. 2002;89:213-228.

4. Borysiewicz LK, Fiander A, Nimako M, et al. A recombinant vaccinia virus encoding human papillomavirus types 16 and 18, E6 and E7 proteins as immunotherapy for cervical cancer. Lancet. 1996;347:1523-1527.

5. Lin KY, Guarnieri FG, Staveley-O'Carroll KF, et al. Treatment of established tumors with a novel vaccine that enhances major histocompatibility class II presentation of tumor antigen. Cancer Res. 1996;56:21-26.

6. Feltkamp MC, Smits HL, Vierboom MP, et al. Vaccination with cytotoxic $\mathrm{T}$ lymphocyte epitope-containing peptide protects against a tumor induced by human papillomavirus type 16-transformed cells. Eur J Immunol. 1993;23:22422249 . 
7. He Z, Wlazlo AP, Kowalczyk DW, et al. Viral recombinant vaccines to the E6 and E7 antigens of HPV-16. Virology. 2000;270:146-161.

8. Smahel M, Sima P, Ludvikova V, et al. Modified HPV16 E7 genes as DNA vaccine against E7-containing oncogenic cells. Virology. 2001;281:231-238.

9. Osen W, Peiler T, Ohlschlager P, et al. A DNA vaccine based on a shuffled E7 oncogene of the human papillomavirus type 16 (HPV 16) induces E7-specific cytotoxic T-cells but lacks transforming activity. Vaccine. 2001;19:4276-4286.

10. Daemen T, Pries F, Bungener L, et al. Genetic immunization against cervical carcinoma: induction of cytotoxic $\mathrm{T}$ lymphocyte activity with a recombinant alphavirus vector expressing human papillomavirus type 16 E6 and E7. Gene Therapy. 2000; 7:1859-1866.

11. Liu DW, Tsao YP, Hsieh CH, et al. Induction of CD8 Tcells by vaccination with recombinant adenovirus expressing human papillomavirus type 16 E5 gene reduces tumor growth. J Virol. 2000;74:9083-9089.

12. Wu TC, Guarnieri FG, Staveley-O'Carroll KF, et al. Engineering an intracellular pathway for major histocompatibility complex class II presentation of antigens. Proc Natl Acad Sci USA. 1995;92:11671-11675.

13. Rodriguez F, Zhang J, Whitton JL. DNA immunization: ubiquitination of a viral protein enhances cytotoxic $\mathrm{T}$ lymphocyte induction and antiviral protection but abrogates antibody induction. $J$ Virol. 1997;71:8497-8503.

14. Pasquini S, Deng H, Reddy ST, et al. The effect of $\mathrm{CpG}$ sequences on the B cell response to a viral glycoprotein encoded by a plasmid vector. Gene Therapy. 1999;6:14481455.

15. Wlazlo AP, Giles-Davis W, Clements A, et al. Generation and characterization of monoclonal antibodies against the E6 and E7 oncoproteins of HPV. Hybridoma. 2001;20:257263.

16. Deng H, Kowalczyk D, O InSug, et al. A modified DNA vaccine to $\mathrm{p} 53$ induces protective immunity to challenge with a chemically induced sarcoma cell line. Cell Immunol. 2002;215:20-31.

17. Kowalczyk DW, Wlazlo AP, Shane $S$, et al. Vaccine regimen for prevention of sexually transmitted infections with human papillomavirus type 16. Vaccine. 2001;19:3583-3590.

18. Fu ZF, Dietzschold B, Schumacher CL, et al. Rabies virus nucleoprotein expressed in and purified from insecT-cells is efficacious as a vaccine. Proc Natl Acad Sci USA. 1991;88:2001-2005.

19. Blaszczyk-Thurin M, Ertl IO, Ertl HC. An experimental vaccine expressing wild-type p53 induces protective immunity against glioblastoma cells with high levels of endogenous p53. Scand J Immunol. 2002;56:361-375.

20. Kowalczyk DW, Wlazlo AP, Blaszczyk-Thurin M, et al. A method that allows easy characterization of tumor-infiltrating lymphocytes. J Immunol Methods. 2001;253:163-175.
21. Xiang Z, Ertl HC. Manipulation of the immune response to a plasmid-encoded viral antigen by coinoculation with plasmids expressing cytokines. Immunity. 1995;2:129-135.

22. Ramshaw IA, Ramsay AJ. The prime-boost strategy: exciting prospects for improved vaccination. Immunol Today. 2000;21:163-165.

23. Yang ZY, Wyatt LS, Kong WP, et al. Overcoming immunity to a viral vaccine by DNA priming before vector boosting. J Virol. 2003;77:799-803.

24. Koutsky LA, Ault KA, Wheeler CM, et al. A controlled trial of a human papillomavirus type 16 vaccine. $N$ Engl $J$ Med. 2002;347:1645-1651.

25. Krieg AM. CpG motifs in bacterial DNA and their immune effects. Annu Rev Immunol. 2002;20:709-760.

26. Katz DH, Hamaoka T, Dorf ME, et al. Cell interactions between histoincompatible $\mathrm{T}$ and $\mathrm{B}$ lymphocytes. IV. Involvement of the immune response (Ir) gene in the control of lymphocyte interactions in responses controlled by the gene. . J Exp Med. 1973;138:734-739.

27. Vierboom MP, Feltkamp MC, Neisig A, et al. Peptide vaccination with an anchor-replaced CTL epitope protects against human papillomavirus type 16-induced tumors expressing the wild-type epitope. $J$ Immunother. 1998;21:399-408.

28. Schirmbeck R, Bohm W, Ando K, et al. Nucleic acid vaccination primes hepatitis $\mathrm{B}$ virus surface antigen-specific cytotoxic $\mathrm{T}$ lymphocytes in nonresponder mice. $J$ Virol. 1995;69:5929-5934.

29. Xiang ZQ, Pasquini S, Ertl HC. Induction of genital immunity by DNA priming and intranasal booster immunization with a replication-defective adenoviral recombinant. J Immunol. 1999;162:6716-6723.

30. Ji H, Wang TL, Chen $\mathrm{CH}$, et al. Targeting human papillomavirus type $16 \mathrm{E} 7$ to the endosomal/lysosomal compartment enhances the antitumor immunity of DNA vaccines against murine human papillomavirus type 16 E7expressing tumors. Hum Gene Therapy. 1999;10:2727-2740.

31. Reyes-Sandoval A, Ertl HC. DNA vaccines. Curr Mol Med. 2001;1:217-243.

32. Gunn GR, Zubair A, Peters C, et al. Two Listeria monocytogenes vaccine vectors that express different molecular forms of human papilloma virus-16 (HPV-16) E7 induce qualitatively different T-cell immunity that correlates with their ability to induce regression of established tumors immortalized by HPV-16. J Immunol. 2001;167:6471-6479.

33. O InSug, Blaszczyk-Thurin M, Shen CT, et al. A DNA vaccine expressing tyrosinase-related protein-2 induces $\mathrm{T}$ cell-mediated protection against mouse glioblastoma. Cancer Gene Therapy. 2003;10:678-688.

34. Kotecha MT, Afghan RK, Vasilikopoulou E, et al. Enhanced tumour growth after DNA vaccination against human papillomavirus virus E7 oncoprotein: evidence for tumour-induced immune deviation. Vaccine. 2003;21:25062515 . 